$\xi=-1$ 罥

\title{
Diagnosis of internal combustion engine based on mechanical noise
}

\author{
Sergei S. Kapitonov ${ }^{1}$, Sergei Y. Grigorovich ${ }^{1}$, Sergei A. Medvedev ${ }^{1}$, Ivan I. Kurbakov ${ }^{1}$ \\ ${ }^{1}$ National Research Mordovia State University, Saransk, Russia Address for correspondence: \\ Sergei S. Kapitonov, 68 Bolshevistskaya Str., Saransk 430005, Republic of Mordovia, Russia \\ *Corresponding author E-mail: kapss88@mail.ru
}

\begin{abstract}
The work of sensors is investigated in the article when they are used to detect malfunctions of automobile engines by the method of analyzing noise spectrum. The method of diagnostics of internal combustion engines for noise and vibration has been developed. This method allows to determine the fault at an early stage in a short period of time (the diagnostic process takes 5-10 minutes). The proposed solution allows to reduce the degree of influence of the human factor. Such an approach to diagnostics makes it possible to determine a huge number of faults due to the use of modern computer technologies. There is no need to dismantle the unit to determine a malfunction, which also contributes to a significant reduction in overall repair time. The model of the hardware part of the complex is based on the developed method for diagnosing engine malfunctions by noise and vibration. Technologies of National Instruments were used to create the layout. The program part is developed in the visual programming environment LabVIEW. The investigation of different types of noise sensors for use in the measuring complex was carried out using the developed hardware and software. Three types of noise sensors were investigated. The conclusion can be drawn based on the results of the study, that a piezoelectric pickup is better suited for diagnosing or analyzing noise. The analysis of the most frequently encountered car faults was carried out with the aim of forming real engine operating modes and testing the model.
\end{abstract}

Keywords: Sensor; Signal Spectrum; Noise; Internal Combustion Engine; Diagnostics; Malfunction; Car; Human Factor; Measuring Complex.

\section{Introduction}

Transport is one of the most important spheres for social and economic life of human today. Most cars that are currently in use are significantly outdated. A lot of cars are idle due to serious breakdowns, leading to significant economic losses.

Reliability, environmental friendliness, economy, safety, performance of a car are determined by the operation of its engine Therefore, timely detection of malfunctions and support of engine performance is an important task. [1]

From year to year, internal combustion engines are improved, which is determined by the complexity of the design and additional elements, that contribute to increasing its capacity. [2], [3] Diagnosis of such a device becomes more difficult and expensive Therefore, search for new diagnostic methods is urgent task, solution of which will allow to determine the malfunctions as simply, quickly and reliably as possible. Thus, it becomes possible to get rid of human factor in determining the malfunction. [4], [5].

Most diagnostic methods are specialized on certain engine nodes. Therefore, many tests and various diagnostic equipment are required in order to make a complete diagnosis. This process takes a lot of time and should be conducted under clear guidance of qualified personnel.

The main drawbacks of existing methods and means of diagnosis is. [6], [7].

1) There is no possibility of determining a malfunction at an early stage.

2) High degree of influence of the human factor.

3) Limited number of faults detected by electronic sensors.
4) In some cases, a complete disassembly of the engine is required to determine the malfunction.

The approach to diagnosis of internal combustion engines based on analysis of information on spectrum of its noise is proposed in the article. This approach greatly simplifies process of detecting a malfunction and eliminates human factor. [8,9]

\section{Description of decision}

It is necessary to determine the type of noise sensors used in the test complex. Let's consider the main types of sensors. Many principles of converting sound vibrations into electrical vibrations exist. Noise sensors are implemented on the basis of each principle.

One of the first noise sensors was coal, which we still use in our phones. Its action is based on a change in resistance between grains of coal powder, when pressure changes on their totality. High sensitivity allows using a carbon microphone without amplifiers. Disadvantages of this microphone are that it is very unstable. Useful electrical signal is generated when the contacts between individual grains of powder break and restore, which causes a lot of noise. Also, significant nonlinear distortions and large nonuniformity of the frequency response arise.

Then electromagnetic sensor appeared. Magnetic resistance of system changes under influence of diaphragm of sound pressure, and consequently also magnetic flux, which is directed through turns of winding, wound on coils of this system. Output signal in such a system is alternating voltage of audio frequency arising at the winding terminals. Electromagnetic sensor has a narrow fre- 
quency range, strong linear distortion, high non-uniformity of the frequency response. It shows itself very stable in work.

Electrodynamic sensor has become very widespread for purpose of sound and sound amplification. These sensors are divided into reel and tape. The main advantages of the sensor are a small uneven frequency response and a fairly wide frequency range.

At present, the capacitor sensor has become most widespread. Sensor works as follows. Capacity of the capacitor changes with active vibrations of the membrane with frequency of sound pressure acting on membrane, so that an alternating current of the same frequency appears in the circuit and voltage, that is the output signal falls on load resistance. Structurally connected to the sensor itself amplifiers having a small gain (of the order of 1), high input and low output impedances are provided for almost all modern capacitor sensors. Condenser sensors have the highest quality indicators, such as wide frequency range, low frequency response irregularity, low nonlinear and transient distortion, high sensitivity and low noise level.

In addition, electret sensors exist, they are similar to the principle of operation with condenser-type microphones. The sensor uses an electret plate as a fixed capacitor plate and a constant output source. Property of these materials to maintain a surface charge for a long time is used. At first, electret sensors were not available to everyone because of the very high price, and a very high output resistance provided an opportunity to realize them only in tube circuits. The appearance of field-effect transistors has led to the appearance of extremely efficient and compact electret sensors, they went in the same housing with a preamplifier in the same case with a field-effect transistor. Electret sensor has high impedance as opposed to dynamic sensors having a low electrical resistance of the coil, which forces them to be connected to amplifiers with high output impedance. Such sensors require an external power source, although there is no need for polarizing voltage.

Piezoelectric sensor exists also, whose operation is based on a piezoelectric effect. Essence of this effect is that positive and negative charges appear on the faces of a piezoelectric crystal under compression or tension, like polarization charges. Working as a pressure sensor made it possible to create the first hydrophones and record ultra-low frequency sounds typical of marine life. Piezoelectric effect is observed in anisotropic dielectrics, usually in crystals of sufficiently low symmetry. Polycrystalline insulators with an ordered structure, ceramic and polymers can also have a piezoelectric effect. Single-crystal quartz, which has high temperature stability of its properties, mechanical strength, low dielectric losses and moisture resistance, is widely distributed among piezoelectrics. The edges of the quartz plate are provided with metal plates in order to use polarization charges appearing on opposite faces when it deforms. Charges equal and opposite in sign polarization are induced on such plates, and an electric current appears in the outer wires connecting the plates.

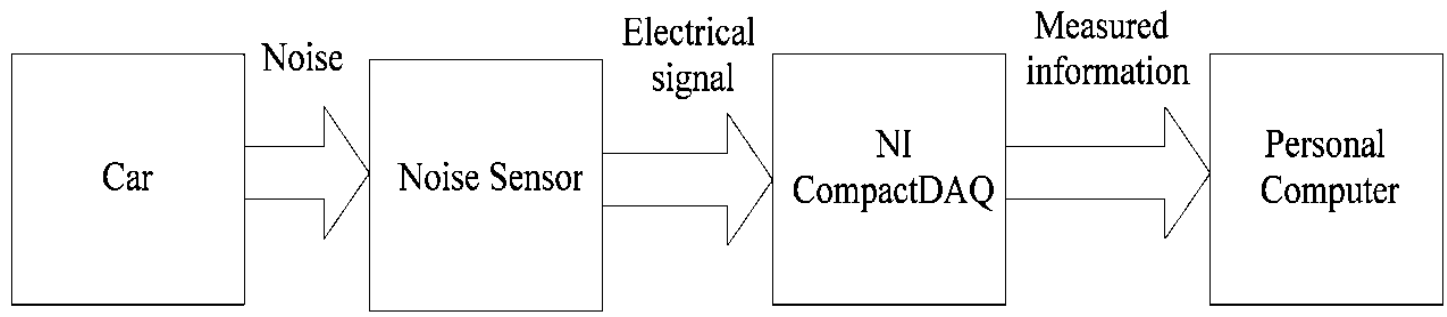

Fig. 1: Approach to Diagnosis of an Internal Combustion Engine.

Piezoelectric properties are more pronounced in crystals of Rochelle salt than in quartz. Due to this, it is used in many piezoelectric devices.

The suggested approach to diagnostics of internal combustion engine faults is presented in Fig. 1. This approach consists of the following steps.

1) Creation of a common database of regularly operating vehicle units and assemblies and a database of possible malfunctions.

2) Obtaining signal spectrum of a faulty unit or unit using noise and vibration sensors

3) Comparison of the obtained spectrum with spectrum of serviceable units and aggregates and determination of the fault in parallel on the basis of information from the noise and vibration sensors.

4) Comparison of the results obtained by examining the signa from the noise and vibration sensors and deciding on the nature of the malfunction.

Process for diagnosing a faulty motor is as follows. Spectral composition of noise emanating from the running engine is fixed by means of a measuring complex and transmitted for processing to a personal computer. The measuring complex consists of a noise sensor interfaced with equipment from National Instruments. CompactDAQ was used to implement the measuring complex. Information processing and decision-making on nature of malfunction occur in the personal computer.

The program is developed in LabVIW to implement processing of information from the measuring complex and to decide on nature of engine malfunction. The main feature of the program is ability to analyze, study and compare spectrum of audio signals measured from different sources of noise. The program can be used both for diagnostics of malfunctions of automobile engines by comparison of a spectrum of serviceable and faulty units, and for definition of malfunctions of various devices. Advantages of this approach to determining engine failures are high accuracy, absence of human factor and low diagnostic time. Spectrum of the measured sound signals, correlated with noise spectrum of serviceable engine, are displayed on front panel of the program. The user himself is able to assess degree of divergence of spectrum of sound signals.

\section{Results of research}

Diagnostics of the engine of the car SKODA Octavia is carried out with purpose of check of working capacity of the offered approach. Measurements were performed using three noise sensors: electret, electrodynamic and piezoelectric. These sensors are chosen to determine the most suitable of them for the solution of the task.

Consider the dependence of output voltage of the sensor on frequency of gasoline engine with a defect. Further, we combine results obtained with noise spectrum of working motor to understand which sensor should be used for the diagnosis of internal combustion engines. The fourth cylinder spark was turned off as a malfunction.

A comparison of the spectrum of a gasoline internal combustion engine of the SKODA Octavia is shown in Figures 2-4 (1 - serviceable engine, 2 - engine with malfunction). 


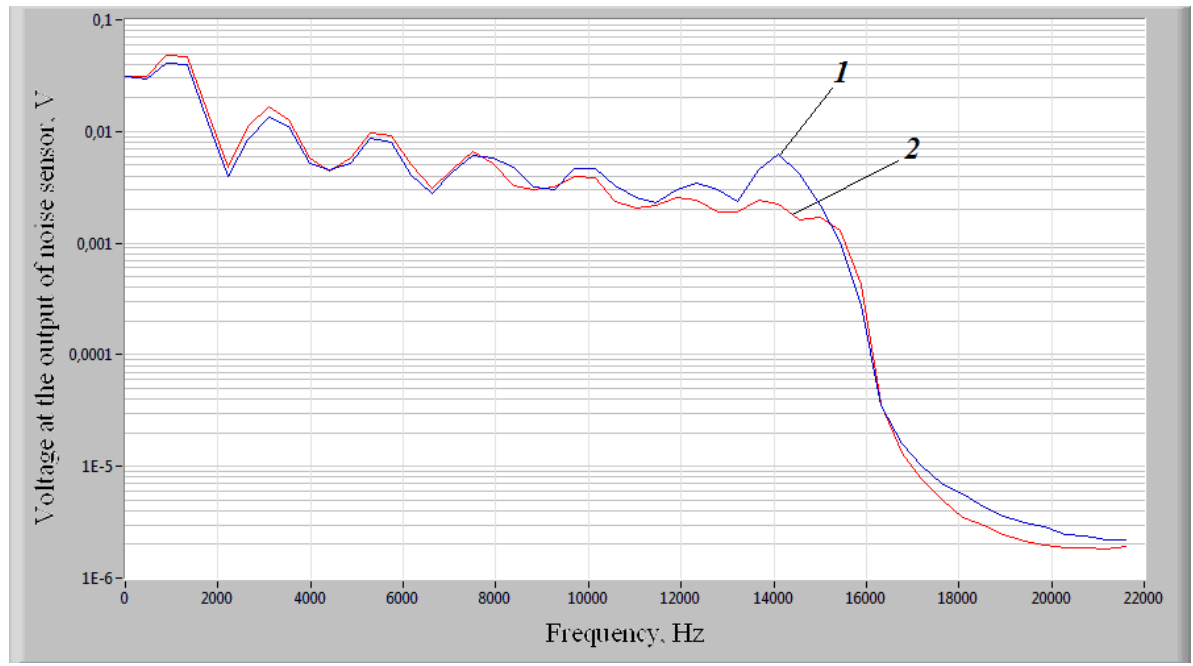

Fig. 2: Dependence of Output Voltage from Frequency of Electret Sensor.

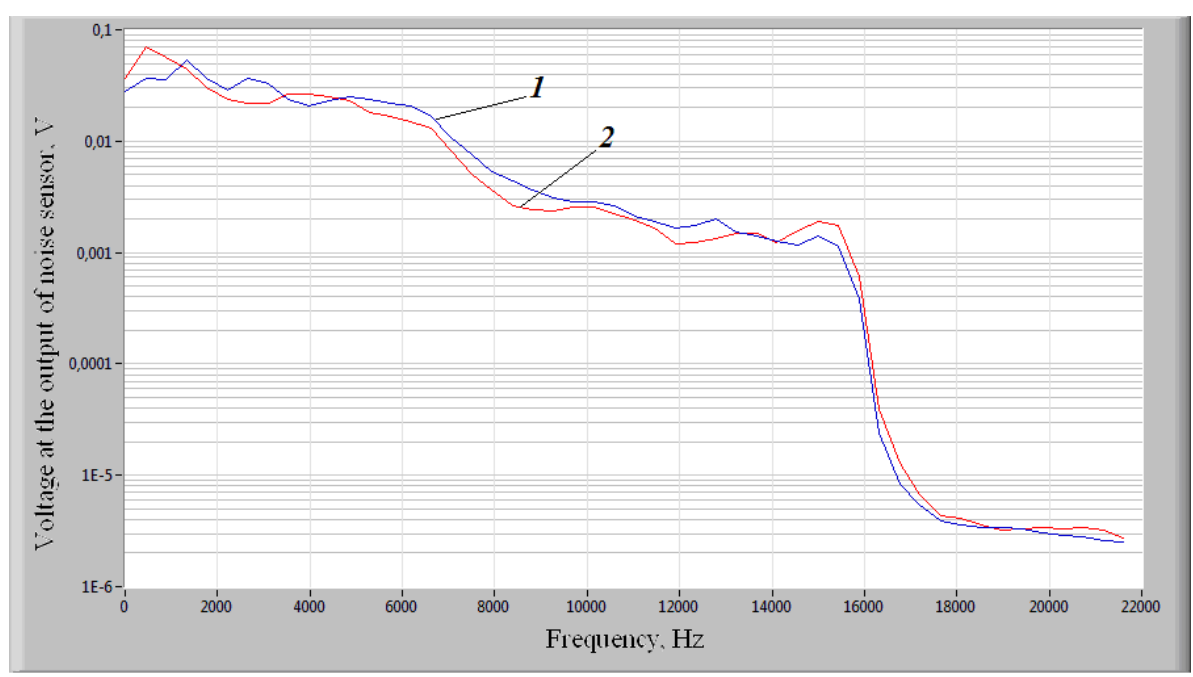

Fig. 3: Dependence of Output Voltage from Frequency of Electrodynamic Sensor.

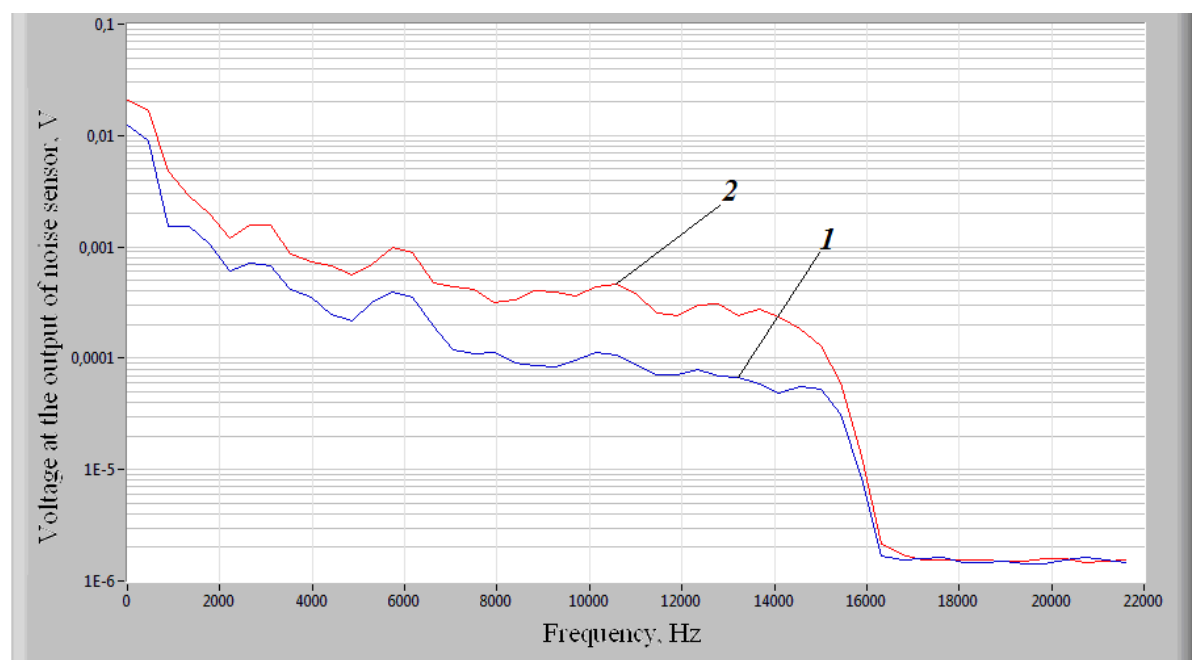

Fig. 4: Dependence of Output Voltage from Frequency of Piezoelectric Sensor.

Jump in output signal at frequencies from $13-15 \mathrm{kHz}$ in operation of a defective motor is observed in Fig. 2. Difference in the noise spectrum of engines is also not easy to see in Fig. 3. Since the spectra are practically identical and have no special expressions. Thus, this fault can not be analyzed with help of electret and electrodynamic sensors, since the noise spectrum remains practically unchanged.

The piezoelectric pickup has excellent sensitivity properties, it interacts with a noise source and experiences shearing deformation. Thanks to this, we can observe in Fig. 4 dependence on which the malfunction can be diagnosed. The spectrum are sym- metrical, the work of the engine with a defect from the serviceable motor is difference in thousandth $\mathrm{mV}$ at frequencies from 0 to 15 $\mathrm{kHz}$. Stroke of piston without detonation at idle makes up this difference at engine operating temperature of $90^{\circ}$.

\section{Conclusions}

Based on results obtained, it can be concluded, that piezoelectric sensor is better suited for diagnosis or analysis of noise, since it 
has a greater sensitivity, and therefore more accurate readings, in contrast to other sensors.

The complex can be applied in the following areas.

1) Enterprises engaged in the production of automotive equipment for testing the quality of assembly.

2) Enterprises engaged in repair and maintenance: cars; trucks agricultural machinery; military equipment.

Developed hardware-software complex for diagnosing car faults by noise will allow:

1) Identify faults at an early stage, not allowing the whole unit to fail;

2) Significantly reduce the degree of influence of the human factor on the process of determining the malfunction;

3) Significantly increase the number of identified faults;

4) Increase the probability of error-free identification of faults;

5) Improve the performance of the diagnostic process

\section{References}

[1] Bespalov N. N., Ilyin M. V., Kapitonov S. S., Grigorovich S. Y Application of computer technologies for investigation of thermal processes in converter of ac drive. 2016 International Siberian Conference on Control and Communications, SIBCON 2016. Moscow, 2016, C. 7491763.

[2] Kapitonov S. S., Bespalov N. N., Il'in M. V., Gulyaev I. V. Selection of the heating method of semiconductor devices during their testing in a high-conductivity state. Russian Electrical Engineering, 2017, V. 88, no. 6, pp. 351-354

[3] Kapitonov S. S., Bespalov N. N., Il'in M. V., Gulyaev I. V. Selection of power semiconductor devices for parallel connection. Russian Electrical Engineering, 2017, V. 88, no. 6, pp. 336-341.

[4] Bespalov N. N., Kapitonov S. S., Ilyin M. V., Lysenkov, A. E. Selection method of power semiconductor devices for serial group circuits of power converters. 2017 International Siberian Conference on Control and Communications, SIBCON 2017. - Astana, 2017. C. 7998523

[5] Bespalov N. N., Lysenkov, A. E., Ilyin M. V., Kapitonov S. S About the influence of parameters values technological fluctuation on the power MOSFET thermal mode. 2017 International Siberian Conference on Control and Communications, SIBCON 2017. Astana, 2017. C. 7998524.

[6] Bespalov N. N., Kapitonov S. S., Ilyin M. V., Evishev A. V., Zorkin A. V., Volkov A. G. Research of operating modes of thyristor converters in DC electric drive with variation of back-EMF in load. Young Researchers in Electrical and Electronic Engineering Conference (2016 ElConRusNW). - St. Petersburg: IEEE, 2016. P. 539-541.

[7] Bespalov N. N., Kapitonov S. S., Ilyin M. V., Evishev A. V., Zorkin A. V., Volkov A. G. Research of operating modes of thyristor converters in DC electric drive with variation of load inductance. Young Researchers in Electrical and Electronic Engineering Conference (2016 ElConRusNW). - St. Petersburg: IEEE, 2016. - P. $542-544$.

[8] Bespalov Nikolai N., Kapitonov Sergei S., Ilyin Michael V., Evishev Alexei V., Volkov Alexander G. Determination of temperature parameters of IGBT, used in converter of AC electric drive. Young Researchers in Electrical and Electronic Engineering Conference (2017 ElConRus). - St. Petersburg: IEEE, 2017. C. 109.

[9] Montazeri-Gh, M. \& Mahmoodi-K, M. J. Cent. South Univ. (2015) 22: 1737. https://doi.org/10.1007/s11771-015-2692-6. 